\title{
Methotrexate Toxicity in Growing Long Bones of Young Rats: A Model for Studying Cancer Chemotherapy-Induced Bone Growth Defects in Children
}

\author{
Chiaming Fan, ${ }^{1,2}$ Kristen R. Georgiou, ${ }^{1,3}$ Tristan J. King, ${ }^{1,3}$ and Cory J. Xian ${ }^{1,2,3}$ \\ ${ }^{1}$ Sansom Institute for Health Research, and School of Pharmacy and Medical Sciences, University of South Australia, City East Campus, \\ GPO Box 2471, Adelaide, SA 5001, Australia \\ ${ }^{2}$ Discipline of Paediatrics, University of Adelaide, Adelaide, SA 5005, Australia \\ ${ }^{3}$ Discipline of Physiology, University of Adelaide, Adelaide, SA 5005, Australia \\ Correspondence should be addressed to Cory J. Xian, cory.xian@unisa.edu.au
}

Received 10 September 2010; Accepted 21 January 2011

Academic Editor: Andrea Vecchione

Copyright () 2011 Chiaming Fan et al. This is an open access article distributed under the Creative Commons Attribution License, which permits unrestricted use, distribution, and reproduction in any medium, provided the original work is properly cited.

The advancement and intensive use of chemotherapy in treating childhood cancers has led to a growing population of young cancer survivors who face increased bone health risks. However, the underlying mechanisms for chemotherapy-induced skeletal defects remain largely unclear. Methotrexate (MTX), the most commonly used antimetabolite in paediatric cancer treatment, is known to cause bone growth defects in children undergoing chemotherapy. Animal studies not only have confirmed the clinical observations but also have increased our understanding of the mechanisms underlying chemotherapy-induced skeletal damage. These models revealed that high-dose MTX can cause growth plate dysfunction, damage osteoprogenitor cells, suppress bone formation, and increase bone resorption and marrow adipogenesis, resulting in overall bone loss. While recent rat studies have shown that antidote folinic acid can reduce MTX damage in the growth plate and bone, future studies should investigate potential adjuvant treatments to reduce chemotherapy-induced skeletal toxicities.

\section{Introduction}

During childhood and adolescence, bone continues to grow until a peak height and peak bone mass are achieved. It is during these periods that children are most vulnerable to interference to skeletal growth, and disturbance to the growing skeleton results from disruption to the processes of endochondral ossification and/or bone remodeling, which may predispose children to earlier onset of skeletal defects. Due to significant advancements and higher survival rate, cancer chemotherapy has been gaining popularity in treatment of paediatric cancers and has become an important risk factor for bone growth defects in paediatric cancer patients. Intensive chemotherapy for childhood cancers has been shown to cause bone growth defects (bone loss, osteopenia, and fractures).

Methotrexate is the most commonly used antimetabolite in childhood oncology, and both clinical and experimental studies have demonstrated methotrexate-induced bone growth impairment. This paper reviews previous studies in which rat models of methotrexate chemotherapy have been used to investigate chemotherapy-induced bone defects, mechanisms of bone growth arrest and bone loss, and recovery potential.

\section{Bone Growth and Regulation}

Bone growth is the process involving fascinating changes in morphology and biochemistry during development and growth, which gradually ceases until adolescence ends. During bone growth in childhood and adolescence, lengthening of long bones depends on the process of endochondral ossification, in which the growth plate cartilage continues to produce calcified cartilage which serves as a template for formation of primary trabecular bone [1]. Growth plate is situated at both ends of long bones, which is composed of 
three distinct zones: the resting, proliferative, and hypertrophic zones. Bone growth begins as progenitor cells at resting zone are activated and enter the cell cycle at the proliferative zone [2] and produce extracellular matrix rich in collagen-II and aggrecan [1]. The hypertrophic chondrocytes secrete matrix rich in collagen-X and direct mineralisation of their surrounding matrix while undergoing apoptosis [3]. Metaphyseal primary bone formation begins as blood vessels invade the mineralised hypertrophic cartilage, which brings in two cell types (osteoblasts and osteoclasts) that remodel the mineralized cartilage to primary woven bone [1]. While osteoclasts resorb the calcified cartilage, osteoblasts penetrate the invaded calcified cartilage and replace it with spongy bone [1]. Bone lengthens as growth plate cartilage continues to grow and is replaced by bone. Longitudinal bone growth is mainly regulated by genetic and hormonal factors such as growth hormone $(\mathrm{GH})$, insulin-like growth factors (IGFs) $[4,5]$, thyroid hormone and glucocorticoids, sex steroids [6-8], fibroblast growth factors (FGF), epidermal growth factor and related ligands [9] transforming growth factor $\beta$ (TGF- $\beta$ ), and bone morphogenic protein (BMP) $[10,11]$. However, environmental factors such as nutrition [12-14] and medical treatments including chemotherapy have also been shown to be important determinants for bone growth in children, influencing the final height and bone mass of an individual.

\section{The Clinical Issue of Methotrexate-Induced Skeletal Defects}

Cancer chemotherapy has been achieving better success in treatment of paediatric cancers, with a survival rate over $80 \%$ in treating acute lymphoblastic leukaemia (ALL) which is the major childhood cancer. Consequently, childhood chemotherapy has become an important risk factor for bone growth defects in paediatric cancer patients. Methotrexate (MTX) is the most widely used antimetabolite in the treatment of childhood cancers and is critical for treating acute lymphoblastic leukemia (ALL) [15]. MTX is a folate antagonist, commonly used at high-doses for the treatment of malignancies $\left(100-1,000 \mathrm{mg} / \mathrm{m}^{2}\right)[16,17]$ and at lower doses (5-25 mg/week) for the treatment of inflammatory diseases such as rheumatoid arthritis (RA) [17, 18]. Frequently reported adverse effects associated with high-dose MTX include nausea, abdominal distress, intestinal mucositis [19], leucopenia, and bone marrow suppression [20]. High-dose MTX has also been shown to have significant damaging effects on bone growth in children [21].

Clinical studies have highlighted osteopenia as a complication for childhood malignancies, characterised by reduced BMD and increased fracture risks [21, 22]. During intensive chemotherapy, children treated with high-dose MTX in combination with corticosteroids showed depressed bone formation and enhanced bone resorption [23, 24]. On the other hand, some studies reported no significant BMD reduction in survivors of ALL [25]. Since ALL treatment regimens are multiple drug combination therapies with or without cranial irradiation, it is difficult to determine the effects of high-dose MTX alone on bone growth. However, high-doses of MTX and corticosteroid together were found to be associated with longitudinal growth arrest $[26,27]$, a high risk of low bone mass and failure for BMD recovery even after discontinuation of treatment [25]. Overall, the use of high-dose MTX in treating paediatric ALL is encouraged; however, early onset of skeletal complications must be monitored.

\section{Mechanistic Studies on MTX Skeletal Defects with Animal Models}

The mechanism of how childhood cancer chemotherapy affects bone growth remains largely unknown. Since all ALL treatment protocols vary not only in dosage but also, route of administration and use of cranial irradiation, it is difficult to distinguish the effects of individual chemotherapeutic agents on bone growth. Hence, many laboratory studies have been conducted which have enabled investigations into the effects and underlying mechanisms of different chemotherapeutic agents alone on bone growth. For example, in a rat chemotherapy model, doxorubicin, an anthracycline antibiotic and cytotoxic (antineoplastic) agent commonly used against various cancers, caused thinning of growth plate, disturbance of chondrocyte columnar arrangement, increased number of fat cells but decreased hematopoietic cellularity in the bone marrow [28]. Similarly, in vitro studies using human bone marrow cells have shown that corticosteriods (commonly used for treating ALL) can significantly suppress osteoblastic activity, resulting in decreased bone formation [29]. Corticosteroid-induced osteopenia/osteoporosis has been confirmed in animal models [30], has been shown to reduce bone mineral density, and is associated with increased fracture risks in children [31]. In this section, animal studies investigating the mechanisms for MTX-induced skeletal damage are reviewed.

4.1. Methotrexate Chemotherapy-Induced Growth Plate Dysfunction. As bone lengthening is the result of endochondral ossification at the growth plate, chemotherapy-induced growth plate damage may impact on bone lengthening. An earlier in vitro study examining effects of chemotherapeutic agents on chondrocyte proliferation observed no effects of MTX on proliferating chondrocytes [32]. In a rat study, MTX at $60 \mathrm{mg} / \mathrm{m}^{2}$ body surface area (injections once weekly given for 8 weeks) was shown to have no effects on proliferating chondrocytes but to cause an increase in hypertrophic zone thickness and number of hypertrophic chondrocytes [33]. More recent animal studies revealed that while longterm low-dose MTX treatment caused no damage to the growth plate, two cycles of high-dose MTX (at $0.75 \mathrm{mg} / \mathrm{kg}$, 5 days on $/ 9$ days off $/ 5$ days on) caused a significant decrease in growth plate height [34] (Figure 1), which was due to the reduction of chondrocyte proliferation (Figure 1) and collagen-II production, as well as the induction of chondrocyte apoptosis possibly through the Fas/FasL death receptor pathway [35] (Figure 1). Due to the growth plate dysfunction, a significant reduction in the thickness of newly 


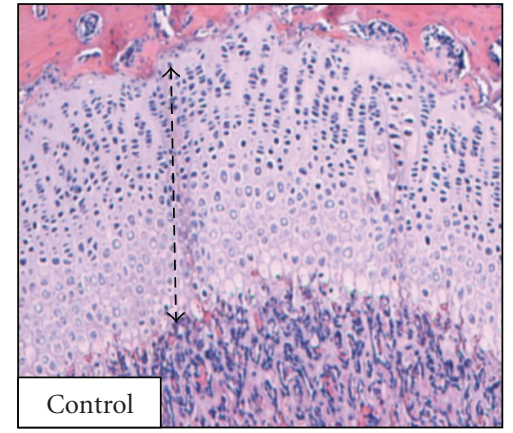

(a)

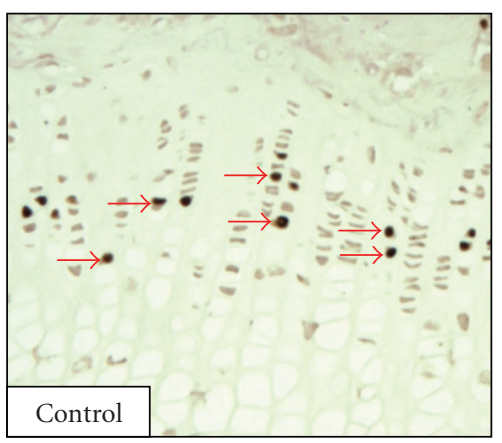

(c)

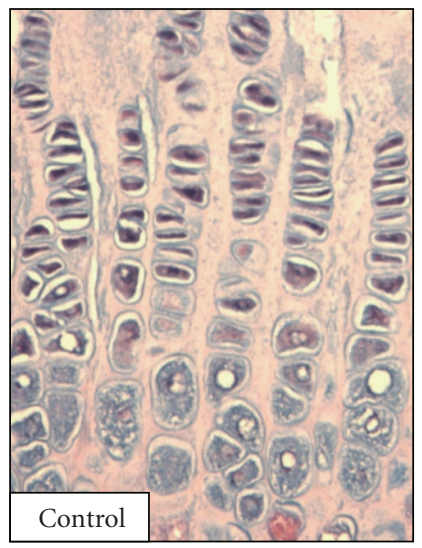

(e)

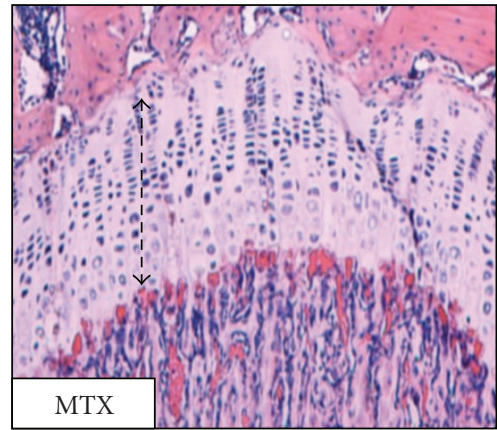

(b)

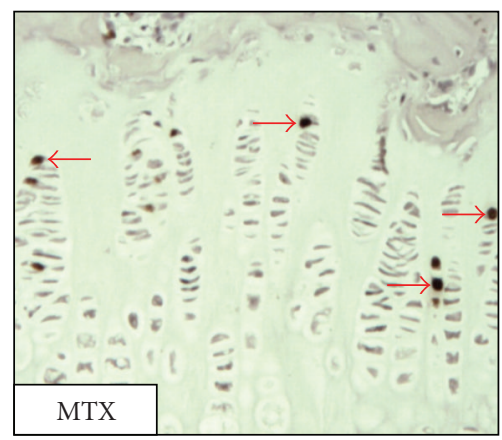

(d)

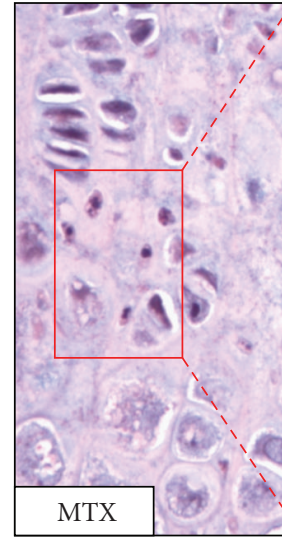

(f)

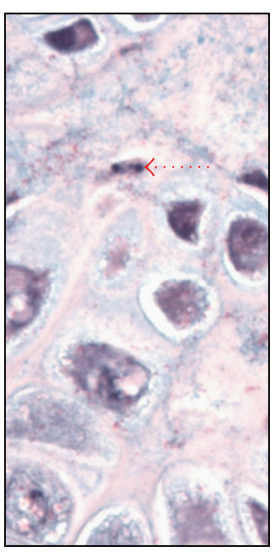

(g)

FIGURE 1: Effect of acute high-dose MTX chemotherapy on growth plate structure and cellular changes in young rats. H \& E stained section of a normal rat tibial growth plate (a) and an MTX-treated rat growth plate (b). Dashed line represents total heights of growth plates. BrdU labeling showing proliferative chondrocytes in a normal rat (c) and an MTX-treated rat (d), with arrows pointing to proliferating chondrocytes. Normal proliferative/hypertrophic chondrocytes of a normal rat (e) showing no apoptosis; MTX-treated rats with apoptotic chondrocytes in lower proliferative/upper hypertrophic zone (f), and a magnified view of apoptotic chondrocyte (g). (Images are from the authors' own lab and have not been published previously.)

formed primary spongiosa bone was also found in the adjacent metaphyseal bone, mirroring the thinning of the growth plate $[35,36]$. These studies suggest that the effect of MTX on growth plate structure and function is largely dependent on the treatment dose and regimen.

4.2. Damaging Effects of Methotrexate Chemotherapy on Osteoblasts, Osteoprogenitors, and Stem Cells. High-dose MTX $\left(100-1000 \mathrm{mg} / \mathrm{m}^{2}\right)$ acts by reversibly inhibiting the enzyme dihydrofolate-reductase (DHFR). DHFR is essential for the synthesis of purine and thymidylate, thus inhibition of DHFR can ultimately inhibit DNA synthesis and therefore cell proliferation [17]. Osteoblast number and function are important indicators of bone formation and bone mass. Earlier studies which analysed the effects of MTX chemotherapy on bone metabolism revealed that MTX has an inhibitory effect on osteoblast function without altering osteoblast numbers in vivo [37]. Another study demonstrated that 


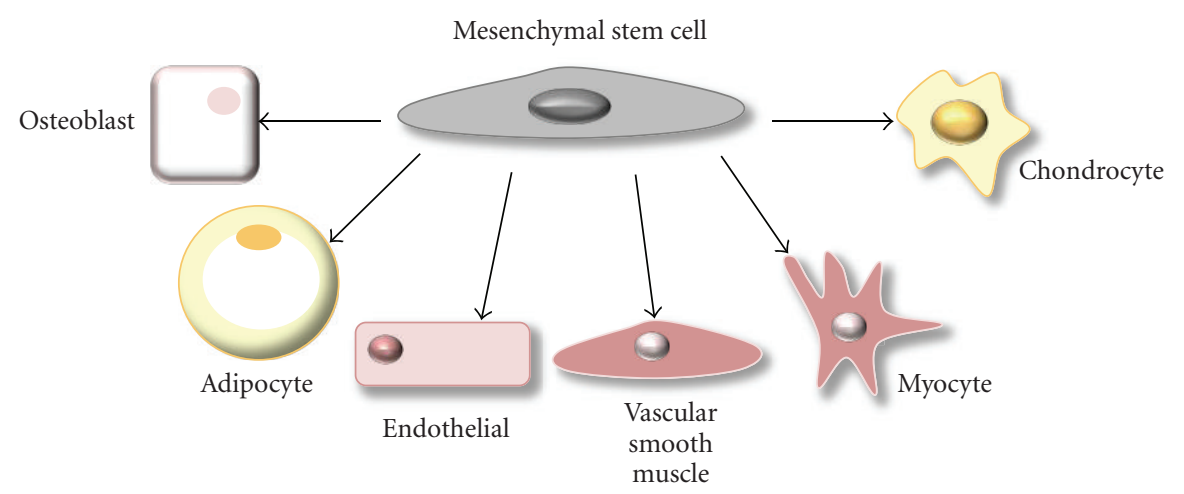

(a)

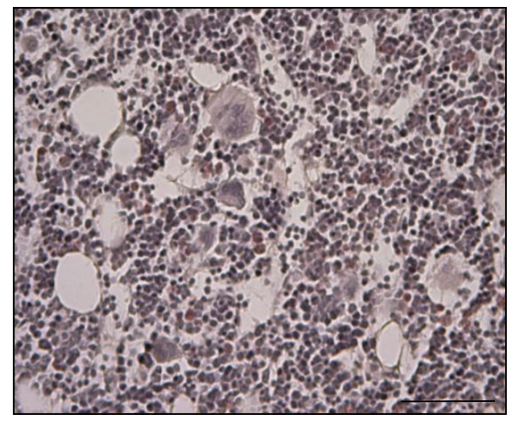

(b)

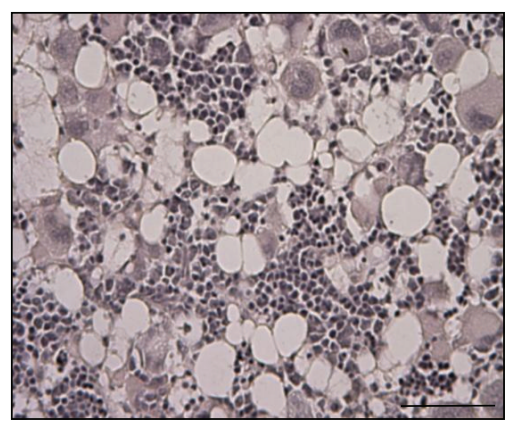

(c)

Figure 2: Mesenchymal stem cell commitment and effects of MTX chemotherapy in bone marrow adiposity. Multipotency of the mesenchymal stem cell (a), illustrated by the capacity to differentiate down a number of cell lineages. H \& E stained bone marrow section taken from a control rat (b) and from an acute high-dose MTX-treated rat (c) showing adipocyte-rich bone marrow. (Images are from the authors' own lab and have not been published previously.)

MTX-induced osteoblastic damage led to a diminished mineralizing surface, mineral apposition rate, and bone formation rate [38]. Consistently, an in vitro study using mouse osteoblasts in culture showed that MTX can decrease osteoblastic cell function in a dose-dependent manner, as indicated by a reduction of matrix calcification and supernatant osteocalcin levels [39]. Although earlier studies found no obvious changes in osteoblast density after MTX treatment in rats, more recent studies reported that both acute high-dose and chronic low-dose MTX treatment in rats can reduce osteoblast density on trabecular bone surface $[34,36]$.

Osteoblasts are derived from stromal progenitor cells or mesenchymal stem cells (MSCs), which can differentiate into osteogenic, chondrogenic, or adipogenic cells [40] (Figure 2). The osteogenic ability of these cells makes it possible to study the toxicity of chemotherapy alone on bone marrow osteoprogenitor cell population and their osteogenic potential. Previously, high-dose chemotherapy (in absence of irradiation) was found to have a direct effect on the stromal population in patients undergoing chemotherapy, caused depletion of bone marrow progenitor cells [41], and resulted in decreased osteoblast differentiation and bone formation $[41,42]$. Recently, rat models of MTX chemotherapy also demonstrated a reduction in bone marrow osteoprogenitor cells and suppressed stromal progenitor cell proliferation $[34,36]$. These in vivo studies suggest that chemotherapy with several drugs in combination or with MTX alone impairs the oestrogenic commitment of the bone marrow progenitor cell population, the severity and recovery of which are major determinants of the extent of bone loss and recovery potential following chemotherapy.

In vitro studies found that human bone marrow MSCs appear more resistant to MTX, cyclophosphomide and busulphan than peripheral blood mononuclear cells [43], and that isolated MSCs from normal or chemotherapyexposed patients remain unaffected by the presence of cytotoxic agents in culture when assessed for differentiation or proliferative potential [44]. These studies suggest that while the already committed osteoprogenitor cells can be more easily affected by chemotherapy drugs, the quiescent MSCs appear to have the capacity to maintain their number and their stem cell character both in vivo and in vitro in response to some chemotherapeutic agents [44]. This perhaps explains why the bone and bone marrow stroma can regenerate in the MTX acute treatment models in rats [34-36].

4.3. Methotrexate Chemotherapy-Induced Marrow Adiposity. In recent rat studies of MTX chemotherapy-induced bone defects, it has been found that apart from the reduced osteoblast number and trabecular bone volume, there is a significant increase in marrow adiposity [35, 36] (Figure 2). 


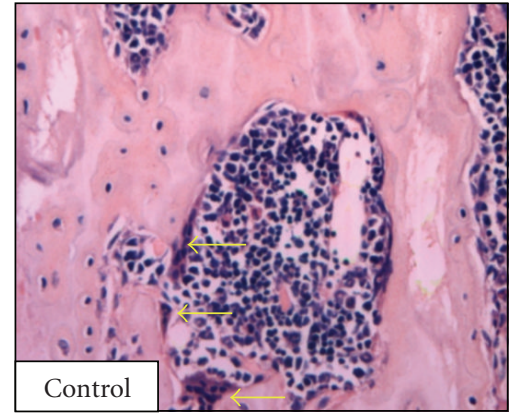

(a)

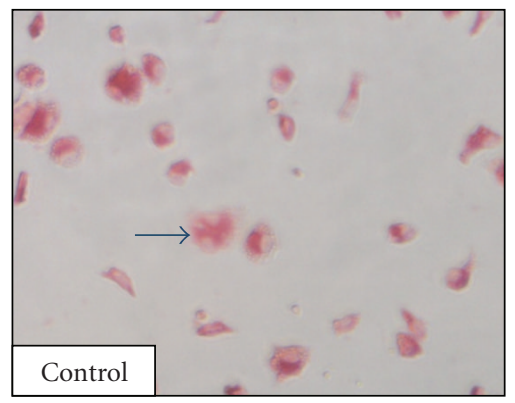

(c)

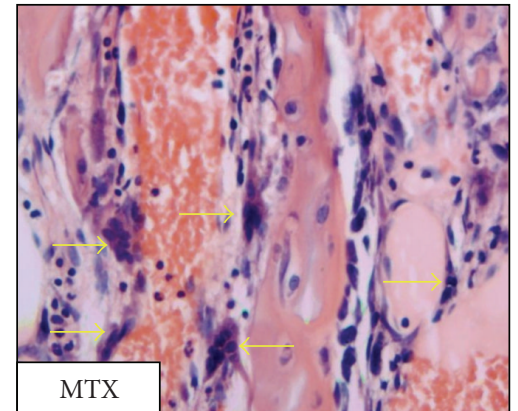

(b)

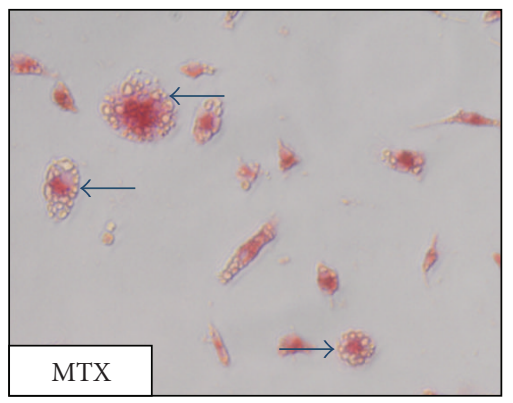

(d)

FIGURE 3: Effect of MTX chemotherapy on osteoclast density in young rats. H \& E stained sections showing osteoclasts along trabecular bone surface in a control rat (a) and an MTX-treated rat (b), with arrows pointing to multinucleated osteoclasts. TRAP-stained osteoclasts formed

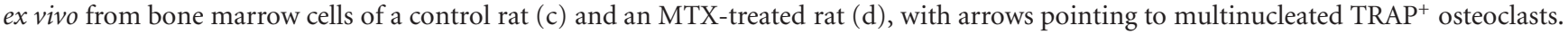
(Images are from the authors' own lab and have not been published previously.)

Consistently, one early in vitro study demonstrated that the presence of MTX can significantly increase the number of fat-containing cells in bone marrow culture [45]. These studies suggest that MTX chemotherapy can cause a reciprocal switch in bone versus fat volume in the bone marrow microenvironment. Since adipocytes and osteoblasts share a common precursor (bone marrow MSC), it has been proposed that bone loss may result from a switch in favour of adipocyte differentiation over osteoblast commitment. Since the Wnt signalling pathway stimulates osteoblast lineage commitment and inhibits adipocyte formation $[46,47]$, it is of interest to examine whether deregulation of Wnt signaling may be involved in this bone/fat reciprocal relationship following MTX chemotherapy.

\subsection{Effects of Methotrexate on Haematopoietic Cells and} Osteoclast Formation. In addition to the damage to osteoblasts, bone marrow stromal cells (discussed above), haematopoietic stem cells (HSCs), and haematopoiesis [48], another possible mechanism for chemotherapy-induced decrease in bone mass in children is the increased formation of bone resorptive cells (osteoclasts) and the alteration to the bone remodeling balance in favour of bone resorption. Clinically, children undertaking high-dose MTX treatment have lower bone mass, with increased urinary and faecal calcium excretion, suggesting increased bone resorption $[24,49]$. Results from both short- and long-term rat studies revealed that MTX can cause an increase in osteoclast density on trabecular bone surface $[34,38,50]$ (Figure 3). Similarly, an increase in the number of empty Howship lacunae on the trabecular surface [51] and excretion of hydroxyproline [50] following MTX administration are evident in animal studies which further support the argument of increased bone resorption. A recent ex vivo study using bone marrow cells obtained from rats treated with MTX showed an increase in the osteoclast precursor cell pool which express surface marker CD $11 b^{+}$and an increase in ex vivo osteoclast formation [34] (Figure 3). Mac-1 (CD11b/CD18) has been shown to play a role in facilitating the differentiation of osteoclast precursors into mature osteoclasts when stimulated by the key osteoclastogenic cytokine RANKL [52]. Collectively, this indicates that MTX chemotherapy affects osteoclastogenesis at the precursor level.

Some clinical data revealed an increased serum level of proinflammatory cytokine TNF- $\alpha$ in patients undergoing chemotherapy [53], suggests a potential role for proinflammatory cytokines in chemotherapy-induced osteoclastogenesis. It is known that, apart from RANKL, osteoclast differentiation and activity can be enhanced by proinflammatory mediators such as IL-1, IL-6, and TNF- $\alpha$ [54]. Whilst increased precursor and mature osteoclast presence within the bone strongly suggests increased resorptive activity, no animal studies have directly investigated this link in chemotherapy model. Future studies are required to investigate the potential role of proinflammatory cytokines in osteoclastogenesis as well as the mechanisms by which 
MTX chemotherapy may induce an inflammatory response within the bone marrow microenvironment.

\section{Conclusions and Future Perspectives}

As longitudinal bone growth occurs during childhood and adolescence, altered bone metabolism during this period may interfere with bone growth and bone mass accrual, which may result in lower peak bone mass, potentially leading to premature onset of osteopenia and increased fracture risk [55]. The advancement and success of chemotherapy in treating childhood cancers (particularly ALL) and thus its increasing use in paediatric oncology have resulted in a growing population of young cancer survivors with increased bone health risks (reduced bone growth and lower peak bone mass). Although the mechanism for chemotherapy-induced bone damage is multifactorial, recent research has revealed that chemotherapeutic agents can directly impair bone growth. In particular, rat studies have confirmed that MTX can directly disrupt the growth plate structure and function by inducing chondrocyte apoptosis. reducing chondrocyte proliferation and cartilage protein synthesis. Dysfunction of the growth plate, therefore, reduces formation of primary woven bone. Direct damage to osteoblasts by decreasing osteoblast activity/formation (possibly through inducing the switch in the bone marrow stromal cells towards adipogenic differentiation at the expense of osteogenesis) and bone marrow osteoprogenitor cells also contributes to reduced bone formation. In addition, MTX chemotherapy has also been shown to increase osteoclast formation and cause aggravated bone resorption, contributing to the associated bone loss.

Given the increased rates of fractures and early onset of osteopenia in childhood survivors of ALL, future studies should investigate strategies to reduce skeletal toxicities and improve quality of life of chemotherapy patients. Currently, recommendations and therapeutic strategies for reducing childhood bone loss during chemotherapy are limited, and there have been few studies investigating potential adjuvant treatments to reduce chemotherapy-induced skeletal toxicities. In this context, the rat models of MTX chemotherapy have also been shown to be useful in demonstrating that folinic acid, an antidote used clinically to reduce toxicity to soft tissues such as gut and bone marrow haematopoietic cells, is also efficacious to reduce or prevent MTX chemotherapy-induced bone growth defects [34].

\section{References}

[1] H. M. Kronenberg, "Developmental regulation of the growth plate," Nature, vol. 423, no. 6937, pp. 332-336, 2003.

[2] D. B. Pateder, R. A. Eliseev, R. J. O'Keefe et al., "The role of autocrine growth factors in radiation damage to the epiphyseal growth plate," Radiation Research, vol. 155, no. 6, pp. 847-857, 2001.

[3] H. Robson, "Bone growth mechanisms and the effects of cytotoxic drugs," Archives of Disease in Childhood, vol. 81, no. 4 , pp. 360-364, 1999.
[4] B. C. J. van der Eerden, M. Karperien, and J. M. Wit, "Systemic and local regulation of the growth plate," Endocrine Reviews, vol. 24, no. 6, pp. 782-801, 2003.

[5] J. Wang, J. Zhou, C. M. Cheng, J. J. Kopchick, and C. A. Bondy, "Evidence supporting dual, IGF-I-independent and IGF-Idependent, roles for $\mathrm{GH}$ in promoting longitudinal bone growth," Journal of Endocrinology, vol. 180, no. 2, pp. 247-255, 2004.

[6] O. Nilsson, D. Chrysis, O. Pajulo et al., "Localization of estrogen receptors- $\alpha$ and $-\beta$ and androgen receptor in the human growth plate at different pubertal stages," Journal of Endocrinology, vol. 177, no. 2, pp. 319-326, 2003.

[7] J. Ophoff, K. Venken, F. Callewaert, S. Boonen, R. Bouillon, and D. Vanderschueren, "Sex steroids during bone growth: a comparative study between mouse models for hypogonadal and senile osteoporosis," Osteoporosis International, vol. 20, no. 10, pp. 1749-1757, 2009.

[8] K. Venken, F. Callewaert, S. Boonen, and D. Vanderschueren, "Sex hormones, their receptors and bone health," Osteoporosis International, vol. 19, no. 11, pp. 1517-1525, 2008.

[9] C. J. Xian, "Roles of epidermal growth factor family in the regulation of postnatal somatic growth," Endocrine Reviews, vol. 28, no. 3, pp. 284-296, 2007.

[10] D. Chen, M. Zhao, and G. R. Mundy, "Bone morphogenetic proteins," Growth Factors, vol. 22, no. 4, pp. 233-241, 2004.

[11] T. A. Linkhart, S. Mohan, and D. J. Baylink, "Growth factors for bone growth and repair: IGF, TGF $\beta$ and BMP," Bone, vol. 19, no. 1, pp. 1S-12S, 1996.

[12] P. Ammann, S. Bourrin, J. P. Bonjour, J. M. Meyer, and R. Rizzoli, "Protein undernutrition-induced bone loss is associated with decreased IGF-I levels and estrogen deficiency," Journal of Bone and Mineral Research, vol. 15, no. 4, pp. 683-690, 2000.

[13] A. L. Bueno and M. A. Czepielewski, "The importance for growth of dietary intake of calcium and vitamin D," Jornal de Pediatria, vol. 84, no. 5, pp. 386-394, 2008.

[14] C. C. Johnston, J. Z. Miller, C. W. Slemenda et al., "Calcium supplementation and increases in bone mineral density in children," The New England Journal of Medicine, vol. 327, no. 2, pp. 82-87, 1992.

[15] C. H. Pui, L. L. Robison, and A. T. Look, "Acute lymphoblastic leukaemia," The Lancet, vol. 371, no. 9617, pp. 1030-1043, 2008.

[16] M. Abromowitch, J. Ochs, C. H. Pui, D. Fairclough, S. B. Murphy, and G. K. Rivera, "Efficacy of high-dose methotrexate in childhood acute lymphocytic leukemia: analysis by contemporary risk classifications," Blood, vol. 71, no. 4, pp. 866-869, 1988.

[17] N. J. Minaur, C. Jefferiss, A. K. Bhalla, and J. N. Beresford, "Methotrexate in the treatment of rheumatoid arthritis. I. In vitro effects on cells of the osteoblast lineage," Rheumatology, vol. 41, no. 7, pp. 735-740, 2002.

[18] B. N. Cronstein, "Low-dose methotrexate: a mainstay in the treatment of rheumatoid arthritis," Pharmacological Reviews, vol. 57, no. 2, pp. 163-172, 2005.

[19] N. Boukhettala, J. Leblond, S. Claeyssens et al., "Methotrexate induces intestinal mucositis and alters gut protein metabolism independently of reduced food intake," American Journal of Physiology, vol. 296, no. 1, pp. E182-E190, 2009.

[20] M. Sosin and S. Handa, "Lesson of the week: low dose methotrexate and bone marrow suppression," British Medical Journal, vol. 326, no. 7383, pp. 266-267, 2003. 
[21] W. Högler, G. Wehl, T. van Staa, B. Meister, A. Klein-Franke, and G. Kropshofer, "Incidence of skeletal complications during treatment of childhood acute lymphoblastic leukemia: comparison of fracture risk with the general practice research database," Pediatric Blood and Cancer, vol. 48, no. 1, pp. 21-27, 2007.

[22] I. M. van der Sluis, M. M. van den Heuvel-Eibrink, K. Hählen, E. P. Krenning, and S. M. P. F. De Muinck Keizer-Schrama, "Altered bone mineral density and body composition, and increased fracture risk in childhood acute lymphoblastic leukemia," Journal of Pediatrics, vol. 141, no. 2, pp. 204-210, 2002.

[23] P. M. Crofton, S. F. Ahmed, J. C. Wade et al., "Effects of intensive chemotherapy on bone and collagen turnover and the growth hormone axis in children with acute lymphoblastic leukemia," Journal of Clinical Endocrinology and Metabolism, vol. 83, no. 9, pp. 3121-3129, 1998.

[24] J. M. Halton, S. A. Atkinson, L. Fraher et al., "Altered mineral metabolism and bone mass in children during treatment for acute lymphoblastic leukemia," Journal of Bone and Mineral Research, vol. 11, no. 11, pp. 1774-1783, 1996.

[25] K. Mandel, S. Atkinson, R. D. Barr, and P. Pencharz, "Skeletal morbidity in childhood acute lymphoblastic leukemia," Journal of Clinical Oncology, vol. 22, no. 7, pp. 1215-1221, 2004.

[26] J. M. Halton, S. A. Atkinson, and R. D. Barr, "Growth and body composition in response to chemotherapy in children with acute lymphoblastic leukemia," International Journal of Cancer, vol. 78, no. 11, pp. 81-84, 1998.

[27] M. B. Viana and M. I. Vilela, "Height deficit during and many years after treatment for acute lymphoblastic leukemia in children: a review," Pediatric Blood and Cancer, vol. 50, no. 2, pp. 509-516, 2008.

[28] B. L. van Leeuwen, W. A. Kamps, R. M. Hartel, R. P. H. Veth, W. J. Sluiter, and H. J. Hoekstra, "Effect of single chemotherapeutic agents on the growing skeleton of the rat," Annals of Oncology, vol. 11, no. 9, pp. 1121-1126, 2000.

[29] S. Walsh, G. R. Jordan, C. Jefferiss, K. Stewart, and J. N. Beresford, "High concentrations of dexamethasone suppress the proliferation but not the differentiation or further maturation of human osteoblast precursors in vitro: relevance to glucocorticoid-induced osteoporosis," Rheumatology, vol. 40, no. 1, pp. 74-83, 2001.

[30] W. S. Jee and W. Yao, "Overview: animal models of osteopenia and osteoporosis," Journal of Musculoskeletal and Neuronal Interactions, vol. 1, no. 3, pp. 193-207, 2001.

[31] M. B. Leonard, "Glucocorticoid-induced osteoporosis in children: impact of the underlying disease," Pediatrics, vol. 119, no. 2, pp. S166-S174, 2007.

[32] H. Robson, E. Anderson, O. B. Eden, O. Isaksson, and S. Shalet, "Chemotherapeutic agents used in the treatment of childhood malignancies have direct effects on growth plate chondrocyte proliferation," Journal of Endocrinology, vol. 157, no. 2, pp. 225-235, 1998.

[33] B. L. van Leeuwen, R. M. Hartel, H. W. B. Jansen, W. A. Kamps, and H. J. Hoekstra, "The effect of chemotherapy on the morphology of the growth plate and metaphysis of the growing skeleton," European Journal of Surgical Oncology, vol. 29, no. 1, pp. 49-58, 2003.

[34] C. Fan, J. C. Cool, M. A. Scherer et al., "Damaging effects of chronic low-dose methotrexate usage on primary bone formation in young rats and potential protective effects of folinic acid supplementary treatment," Bone, vol. 44, no. 1, pp. 61-70, 2009.
[35] C. J. Xian, J. C. Cool, M. A. Scherer et al., "Cellular mechanisms for methotrexate chemotherapy-induced bone growth defects," Bone, vol. 41, no. 5, pp. 842-850, 2007.

[36] C. J. Xian, J. C. Cool, M. A. Scherer, C. Fan, and B. K. Foster, "Folinic acid attenuates methotrexate chemotherapy-induced damages on bone growth mechanisms and pools of bone marrow stromal cells," Journal of Cellular Physiology, vol. 214, no. 3, pp. 777-785, 2008.

[37] O. S. Nilsson, F. C. H. Bauer, L. A. Brostrom, and U. Nilsonne, "Effect of the antineoplastic agent methotrexate on experimental heterotopic new bone formation in rats," Cancer Research, vol. 44, no. 4, pp. 1653-1656, 1984.

[38] D. L. Wheeler, R. A. Vander Griend, T. J. Wronski, G. J. Miller, E. E. Keith, and J. E. Graves, "The short- and long-term effects of methotrexate on the rat skeleton," Bone, vol. 16, no. 2, pp. 215-221, 1995.

[39] K. P. May, D. Mercill, M. T. McDermott, and S. G. West, "The effect of methotrexate on mouse bone cells in culture," Arthritis and Rheumatism, vol. 39, no. 3, pp. 489-494, 1996.

[40] D. Benayahu, "The osteogenic compartment of bone marrow: cell biology and clinical application," Hematology, vol. 4, no. 5, pp. 427-435, 2000.

[41] A. Banfi, M. Podestà, L. Fazzuoli et al., "High-dose chemotherapy shows a dose-dependent toxicity to bone marrow osteoprogenitors: a mechanism for post-bone marrow transplantation osteopenia," Cancer, vol. 92, no. 9, pp. 2419-2428, 2001.

[42] J. H. Davies, B. A. J. Evans, M. E. M. Jenney, and J. W. Gregory, "In vitro effects of combination chemotherapy on osteoblasts: implications for osteopenia in childhood malignancy," Bone, vol. 31, no. 2, pp. 319-326, 2002.

[43] J. Li, H. K. W. Law, L. L. Yu, and G. C. F. Chan, "Differential damage and recovery of human mesenchymal stem cells after exposure to chemotherapeutic agents," British Journal of Haematology, vol. 127, no. 3, pp. 326-334, 2004.

[44] L. P. Mueller, J. Luetzkendorf, T. Mueller, K. Reichelt, H. Simon, and H. J. Schmoll, "Presence of mesenchymal stem cells in human bone marrow after exposure to chemotherapy: evidence of resistance to apoptosis induction," Stem Cells, vol. 24, no. 12, pp. 2753-2765, 2006.

[45] S. P. Hauser, K. B. Udupa, and D. A. Lipschitz, "Murine marrow stromal response to myelotoxic agents in vitro," British Journal of Haematology, vol. 95, no. 4, pp. 596-604, 1996.

[46] C. N. Bennett, K. A. Longo, W. S. Wright et al., "Regulation of osteoblastogenesis and bone mass by Wnt10b," Proceedings of the National Academy of Sciences of the United States of America, vol. 102, no. 9, pp. 3324-3329, 2005.

[47] C. E. Macsai, B. K. Foster, and C. J. Xian, "Roles of Wnt signalling in bone growth, remodelling, skeletal disorders and fracture repair," Journal of Cellular Physiology, vol. 215, no. 3, pp. 578-587, 2008.

[48] K. R. Georgiou, B. K. Foster, and C. J. Xian, "Damage and recovery of the bone marrow microenvironment induced by cancer chemotherapy-potential regulatory role of chemokine CXCL12/receptor CXCR4 signalling," Current Molecular Medicine, vol. 10, no. 5, pp. 440-453, 2010.

[49] M. Nesbit, W. Krivit, R. Heyn, and H. Sharp, "Acute and chronic effects of methotrexate on hepatic, pulmonary, and skeletal systems," Cancer, vol. 37, supplement 2, pp. 10481057, 1976.

[50] K. P. May, S. G. West, M. T. McDermott, and W. E. Huffer, "The effect of low-dose methotrexate on bone metabolism and histomorphometry in rats," Arthritis and Rheumatism, vol. 37, no. 2, pp. 201-206, 1994. 
[51] G. E. Friedlaender, R. B. Tross, and A. C. Doganis, "Effects of chemotherapeutic agents on bone. I. Short-term methotrexate and doxorubicin (adriamycin) treatment in a rat model," Journal of Bone and Joint Surgery. American, vol. 66, no. 4, pp. 602-607, 1984.

[52] H. Hayashi, K. I. Nakahama, T. Sato et al., "The role of Mac-1 (CD11b/CD18) in osteoclast differentiation induced by receptor activator of nuclear factor- $\kappa \mathrm{B}$ ligand," FEBS Letters, vol. 582, no. 21-22, pp. 3243-3248, 2008.

[53] M. Darst, M. Al-Hassani, T. Li et al., "Augmentation of chemotherapy-induced cytokine production by expression of the platelet-activating factor receptor in a human epithelial carcinoma cell line," Journal of Immunology, vol. 172, no. 10, pp. 6330-6335, 2004.

[54] W. J. Boyle, W. S. Simonet, and D. L. Lacey, "Osteoclast differentiation and activation," Nature, vol. 423, no. 6937, pp. 337-342, 2003.

[55] E. M. Dennisona, C. Coopera, and Z. A. Colea, "Early development and osteoporosis and bone health," Journal of Developmental Origins of Health and Disease, vol. 1, pp. 142-149, 2010. 


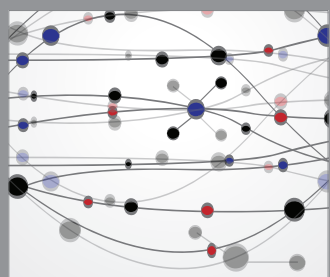

The Scientific World Journal
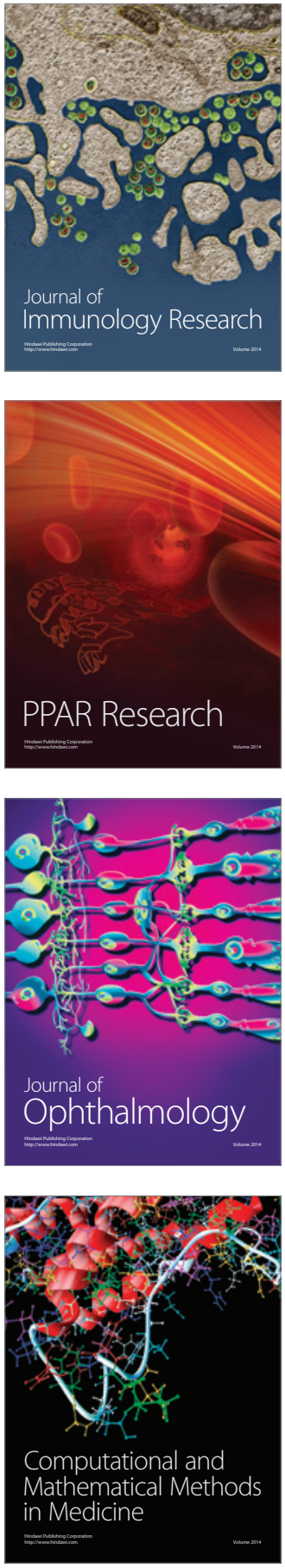

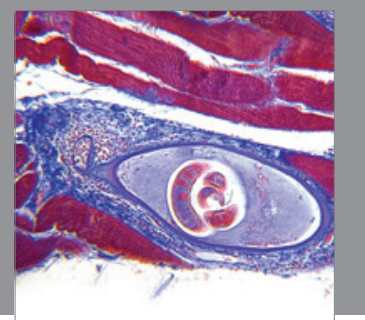

Gastroenterology

Research and Practice
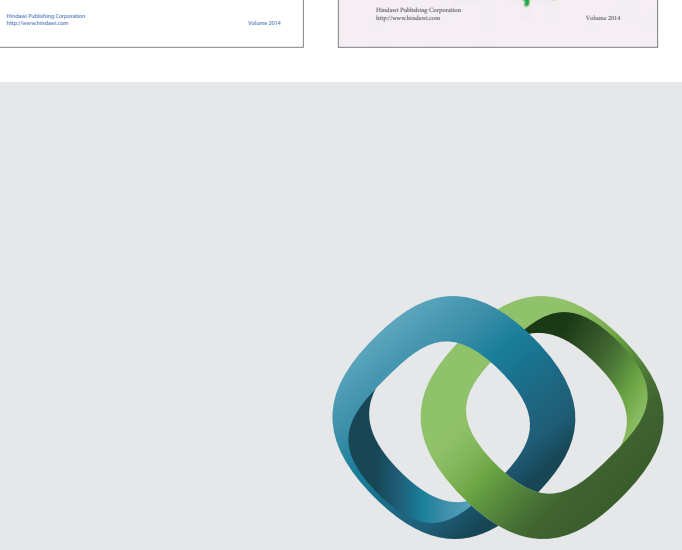

\section{Hindawi}

Submit your manuscripts at

http://www.hindawi.com
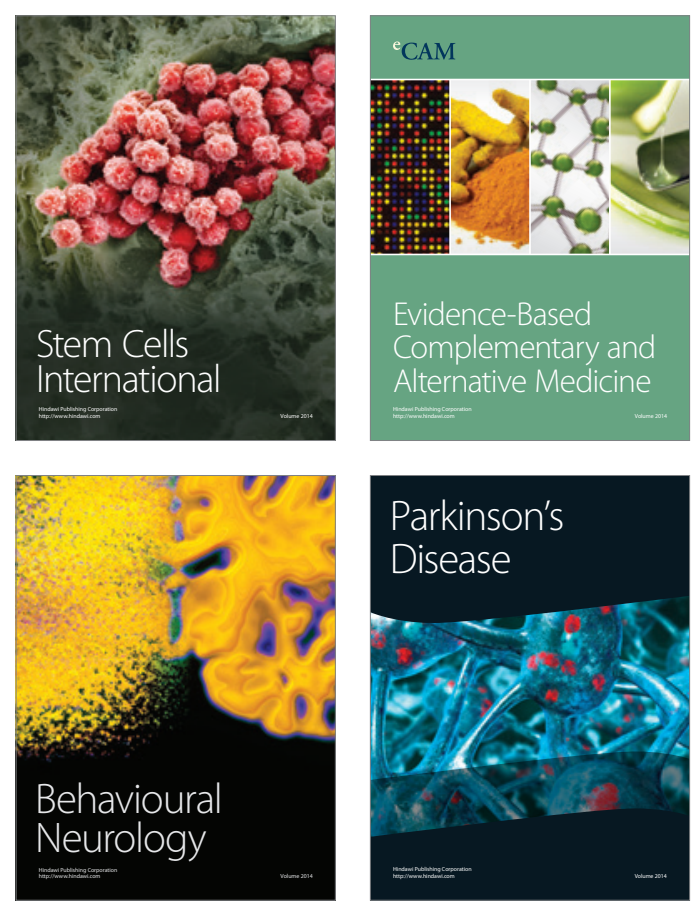

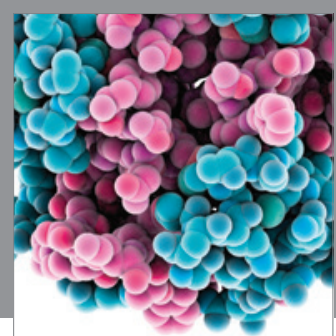

Journal of
Diabetes Research

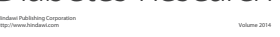

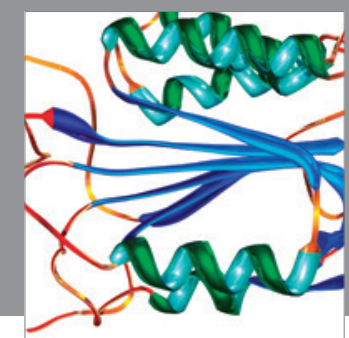

Disease Markers
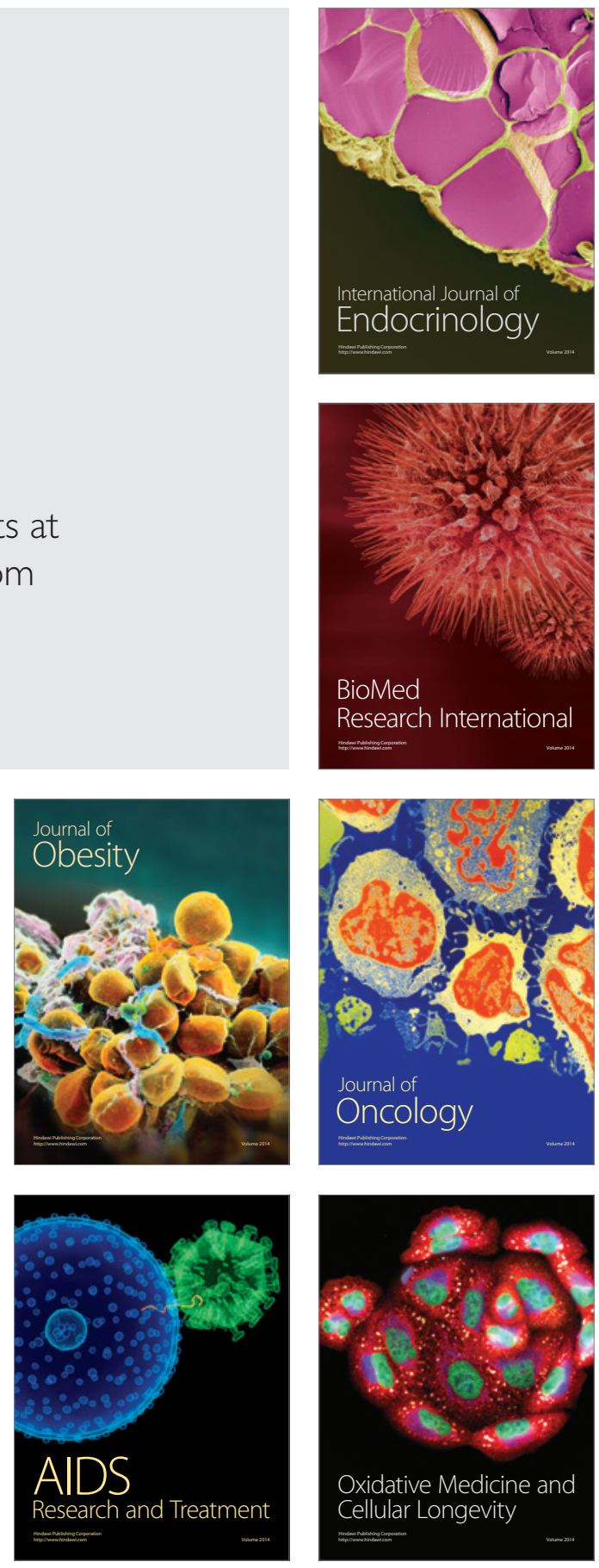